\title{
Study on Measurement Level of Financial Agglomeration Level of Prefecture Level Cities in Guizhou Based on Grey Relational Projection Method
}

\begin{abstract}
Li Dong Mu Zhang
School of Finance, Guizhou University of Finance and Economics, Guiyang Guizhou 550025, China

Abstract

Based on the grey system theory and vector projection principle, this paper studied on the financial agglomeration level evaluation of the nine prefectures in Guizhou Province, which established the evaluation index system and adopted the grey relation projection method. The grey relation projection model takes these prefectures and the corresponding indexes as vectors which was projected to the same vector-----the best decision scheme. According to the size of the projection value, the level of financial agglomeration in each area is determined.
\end{abstract}

\section{Keywords}

Grey system theory; Vector projection principle; Financial agglomeration level

\section{基于灰色关联投影法的贵州地级市金融 集聚水平测度研究}

董礼 张目

贵州财经大学金融学院，贵阳 550025，中国

摘要：本文基于灰色系统理论和矢量投影原理，通过建立评价指标体系，采用灰色关联投影 法，对贵州省九个地州市的金融集聚水平进行评价研究。其中建立的灰色关联投影模型将各 地州市及相应指标视为矢量，分别向同一矢量（最佳决策方案）进行投影，根据投影值的大 小确定各地区金融集聚水平。

关键词: 灰色系统理论; 矢量投影原理; 金融集聚水平

1. 引言

贵州省是一个多民族的省份，又是 一个经济不发达的省份。在改革开放之
后，贵州省经济发展很快。而贵州省经 济的快速发展离不开的金融支撑。因为 金融业将各生产部门的资源进行了有 效的配置 ${ }^{[1]}$ 。但是贵州省各地区的金融 
集聚度相对其他省份而言任然较小。因 此，如何客观评价金融业的集聚程度， 有效地进行产业资源的分配，是金融集 聚程度研究的首要问题。

目前已经有许多关于金融集聚度 的研究和方法, 但目前尚没有关于金融 集聚程度的评估的可靠而统一的标准， 对其研究还处于探索的阶段, 因此, 在 研究方法和理论上还有待于进一步发 展。另外，由于缺乏金融集聚度评价的 标准，没有一个最佳决策方案的参考系， 用这些方法只能对金融集聚度相对的 评价。尚不能客观反映金融集聚程度的 真实情况。因此，研究金融产业集聚问 题无论是从学术价值还是从实践需求 都具有十分重要的积极意义。

\section{2. 国内外文献回顾}

金融集聚程度的评价研究对区域 经济的发展具有重要意义。它可以通过 外部规模经济效益、技术创新、金融外 溢效应、提高金融资源使用效率和自我 强化机制效益等途径对实体经济产生 影响 ${ }^{[2]}$ 。

金德尔伯格 (Kindle-Berger, 1974) ${ }^{[3]}$ 认为银行集聚将带来企业跨地区支付 效率和金融资源跨地区配置效率的提 高。施蒂格勒 (George J. Stigler, 1989) ${ }^{[4]}$ 认为企业集群加强了企业间信息的流 动并增强了企业间的相互信任, 进而使 得企业之间签订专业化上下游产业链 条之间的合约变得容易。费尔德曼

(Feldman, 1999) ${ }^{[5]}$ 的研究表明, 知识 技术的外溢效应随空间距离的扩大而 下降, 集群所带来的知识溢出有利于集 群内经验共享。波松 (Bossone, 2003) ${ }^{[6]}$ 的研究表明, 地理位置的接近有利于 掌握更加丰富的金融信息, 使得银行与 借贷经营的企业家之间信息交流充，从 而提高了整个价值链的利润。在国内研 究学者中, 罗子嫄, 何宜庆等 $(2013)^{[7]}$ 通过构建华东地区金融集聚与经济发
展的耦合关系评价指标体系, 运用熵权 法和耦合模型对 2007-2011 年华东地区 6 省 1 市金融集聚与经济发展的耦合协 调关系进行了综合评价。李静, 白江 (2014) ${ }^{[8]}$ 选取具有代表性的指标构建 了金融集聚的衡量指标体系，运用因子 分析方法计算各省市的分行业集聚度。 邓薇, 吕勇斌等 (2015) ${ }^{[9]}$ 构建了区域金 融集聚评价指标体系, 利用纵横向拉开 档次法对十一五时期 31 个省的金融集 聚程度进行测算和纵横向的双向比较。

\section{3. 灰色关联投影模型的建立}

\section{1. 灰色关联投影法的基本原理}

灰色关联投影法是从矢量投影的 角度探讨多目标决策与评价的新方法。 该方法的每一个决策方案本身就是因 素指标集的映射, 因素指标集一旦确定, 决策方案就得以确定。灰色关联投影法 以各方案在理想方案上的投影值大小, 作为评判决策方案优劣的标准, 并通过 特定的算法, 使重要指标的加权系数得 到进一步加强, 从而全面准确地反映了 各决策方案和理想方案之间的接近程 度。

\section{2. 灰色关联投影模型的建立}

（1）构造多指标决策域集合与因 素指标集合, 然后找出最佳决策方案集 $A_{0}$ 的因素指标集 $V_{0}$ 的属性值 $Y_{0 j}$ 。最后 列出多指标决策域集合 $A$ 对因素指标 集 $V$ 的决策矩阵 $Y$ 。设多指标决策域集 合 $A=\{$ 方案 $1 \cdots$ 方案 $\mathrm{n}\}=\left\{A_{1} \cdots A_{n}\right\}$ 。因素 指标集合 $V=\{$ 指标 $1 \cdots$ 指标 $\mathrm{m}\}=$ $\left\{M_{1}, M_{2} \cdots M_{m}\right\}$ 。记决策方案 $A_{i}$ 对指标 $M_{j}$ 的属性值记为 $Y_{i j}(i=1,2 \cdots n ; j=1$, 
$2 \cdots m$ )。构建方案集 $A$ （包括最佳决 策方案集 $A_{0}$ ）对指标集 $V$ 的属性值矩 阵 $Y$ 。有: $Y=\left(Y_{i j}\right)_{(n+1) m}, \quad(i=0,1,2 \cdots n$ $; j=1,2 \cdots m)$

$$
\text { 既 } Y=\left(\begin{array}{cccc}
Y_{01} & Y_{02} & \cdots & Y_{0 \mathrm{~m}} \\
Y_{11} & Y_{12} & \cdots & Y_{1 \mathrm{~m}} \\
\vdots & \vdots & \ddots & \vdots \\
Y_{\mathrm{n} 1} & Y_{\mathrm{n} 2} & \cdots & Y_{\mathrm{nm}}
\end{array}\right) \text { 由 }
$$

(2) 数据初值化处理和关联度的 计算。为了消除量纲和量纲单位不同所 带来的不可公度性, 决策之前首先应将 评价指标进行无量纲化处理。所谓的无 量纲化处理是对一个数列的所有数据 均除以它的第一个数据。因此该数据列 有共同的起点, 无量纲, 其数据大于零。 对决策矩阵 $Y=\left(Y_{i j}\right)_{(n+1) m}$, $(i=0,1,2, \cdots, n ; j=1,2, \cdots m)$ 进行初值化 生成 $B$ 。 $B=\left(B_{i j}\right)_{(n+1) m}$ 。

显然, 对于理想方案有 $B_{0 j}=1$, $(j=1,2 \cdots m)$ 。由于每一个方案是由 $m$ 个因素指标所决定的, 所以它就构成了 $m$ 维因素指标空间 $V$ 中的一个离散的 方案点, 进行多目标决策就是比较空间 $V$ 中各方案点与理想方案点的关联度。 以 $B_{0 j}(j=1,2, \cdots m)$ 为母因素, 以 $B_{i j}(i=1 \cdots n ; j=1,2 \cdots m)$ 为子因素, 就可以得到其他方案与理想方案的关 联度。记 $R_{i j}$ 为子因素 $B_{i j}(i=1,2, \cdots n ; j=1,2, \cdots m)$ 关于母 因素 $B_{0 j}=1,(j=1,2, \cdots m)$ 的关联度, 则有:

$$
\mathrm{R}_{\mathrm{ij}}=\frac{\min \min \left|B_{0 j}-B_{i j}\right|+\lambda \max \max \left|B_{0 j}-B_{i j}\right|}{\left|B_{0 j}-B_{i j}\right|+\lambda \max \max \left|B_{0 j}-B_{i j}\right|}
$$

通常 $\lambda=0.5$ 。
（3）构造灰色关联度判断矩阵。 $(n+1) \times m$ 个 $R$ 组成的矩阵为多目标灰 色关联度判断矩阵 $F$ 。则有:

$$
\begin{aligned}
F & =\left(\begin{array}{cccc}
R_{01} & R_{02} & \cdots & R_{0 \mathrm{~m}} \\
R_{11} & R_{12} & \cdots & R_{1 \mathrm{~m}} \\
\vdots & \vdots & \ddots & \vdots \\
R_{\mathrm{n} 1} & R_{\mathrm{n} 2} & \cdots & R_{\mathrm{nm}}
\end{array}\right) \\
& =\left(\begin{array}{cccc}
1 & 1 & \cdots & 1 \\
R_{11} & R_{12} & \cdots & R_{1 \mathrm{~m}} \\
\vdots & \vdots & \ddots & \vdots \\
R_{\mathrm{n} 1} & R_{\mathrm{n} 2} & \cdots & R_{\mathrm{nm}}
\end{array}\right)
\end{aligned}
$$

（4）确定各因素指标的权重系数 及加权关联决策矩阵。在研究多指标因 素的影响评价中, 由于各指标因素对方 案的影响程度不同, 所以确定各指标的 权重系数是非常关键的环节。基于各种 因数的考虑, 本文采用熵值赋权法对各 指标进行赋权。根据各项指标观测值所 提供的信息的大小来确定指标权重。对 于 $m$ 个待评方案和 $n$ 项评价指标, 形成 原 始 指 标 数 据 矩 阵 $X=\left(X_{i j}\right)_{n \times m}(i=1,2, \cdots n ; j=1,2, \cdots m)$ 对于某项指标 $X_{j}$, 指标值 $X_{i j}$ 的差距越 大，则该指标在综合评价中所起的作用 就越大; 若某个指标的值全相等, 则给 指标在综合评价中不起作用。设 $X$ 为数 据指标，则有:

$X=\left(\begin{array}{cccc}X_{11} & X_{12} & \cdots & X_{1 \mathrm{~m}} \\ X_{21} & X_{22} & \cdots & X_{2 \mathrm{~m}} \\ \vdots & \vdots & \ddots & \vdots \\ X_{\mathrm{n} 1} & X_{\mathrm{n} 2} & \cdots & X_{\mathrm{nm}}\end{array}\right)$ 其中 $X_{i j}$

为第 $i$ 个方案对 $j$ 个指标的数值。由于 熵值法计算采用的是各个方案某一指 标占同一指标总和的比值, 因此不存在 量纲的影响, 不需要进行标准化处理。 
由于数据中没有负数, 所以本文无需进 行非负化处理。

由 $P_{\mathrm{ij}}=\frac{X_{\mathrm{ij}}}{\sum_{\mathrm{i}=1}^{\mathrm{n}} \mathrm{X}_{\mathrm{ij}}}$ 计算出第 $i$ 个方案第

$j$ 项指标的比重; $\mathrm{e}_{\mathrm{j}}=-\mathrm{k} \sum_{\mathrm{i}=1}^{\mathrm{n}} P_{\mathrm{ij}} \ln P_{\mathrm{ij}}$ 计 算出第 $j$ 个指标的信息熵; 再由 $\mathrm{d}_{\mathrm{j}}=1-\mathrm{e}_{\mathrm{j}}$ 计算出信息熵冗余度; 最后由 $\mathrm{w}_{\mathrm{j}}=\frac{\mathrm{d}_{\mathrm{j}}}{\sum_{\mathrm{j}=1}^{\mathrm{m}} \mathrm{d}_{\mathrm{j}}}$ 计算出第 $j$ 个指标权重。通 常 $\mathrm{k}=\frac{1}{\ln \mathrm{n}}$ 。再由 $C=F \times W$ 确定加权灰 色关联决策矩阵。其中 $W=\left(\mathrm{w}_{1}, \mathrm{w}_{2}, \cdots \mathrm{w}_{\mathrm{j}}, \cdots \mathrm{w}_{\mathrm{m}}\right)$ 。则有:

$C=\left(\begin{array}{cccc}1 & 1 & \cdots & 1 \\ R_{11} & R_{12} & \cdots & R_{1 \mathrm{~m}} \\ \vdots & \vdots & \ddots & \vdots \\ R_{\mathrm{m} 1} & R_{\mathrm{n} 2} & \cdots & R_{\mathrm{nm}}\end{array}\right) \times\left(\begin{array}{c}\mathrm{w}_{1} \\ \mathrm{w}_{2} \\ \vdots \\ \mathrm{w}_{\mathrm{m}}\end{array}\right)$

$=\left(\begin{array}{cccc}\mathrm{w}_{1} & \mathrm{w}_{2} & \cdots & \mathrm{w}_{\mathrm{m}} \\ \mathrm{w}_{1} R_{11} & \mathrm{w}_{2} R_{12} & \cdots & \mathrm{w}_{\mathrm{m}} R_{1 \mathrm{~m}} \\ \vdots & \vdots & \ddots & \vdots \\ \mathrm{w}_{1} R_{\mathrm{n} 1} & \mathrm{w}_{2} R_{\mathrm{n} 2} & \cdots & \mathrm{w}_{\mathrm{m}} R_{\mathrm{nm}}\end{array}\right)$

（5）各个决策方案 $A_{i}$ 在理想方案 $A_{0}$ 上的投影值。将每个决策方案看成 一个行向量（矢量），则称每个决策方 案 $A_{i}$ 与理想方案 $A_{0}$ 之间的夹角 $\theta$ 为灰 色关联投影角。夹角余弦越大, 表明决 策方案 $A_{i}$ 与理想方案 $A_{0}$ 之间的变化方 向越一致。夹角余弦值为:

$$
\begin{aligned}
\cos \theta_{\mathrm{i}} & =\frac{A_{\mathrm{i}} \times A_{0}}{\left\|A_{\mathrm{i}}\right\| \times\left\|A_{0}\right\|} \\
& =\frac{\sum_{\mathrm{j}=1}^{\mathrm{m}} \mathrm{w}_{\mathrm{j}} \times R_{\mathrm{ij}} \times \mathrm{w}_{\mathrm{j}}}{\sqrt{\sum_{\mathrm{j}=1}^{\mathrm{m}}\left(\mathrm{w}_{\mathrm{j}} R_{\mathrm{ij}}\right)^{2}} \times \sqrt{\sum_{\mathrm{j}=1}^{\mathrm{m}} \mathrm{w}_{\mathrm{j}}^{2}}} \\
& =\frac{\sum_{\mathrm{j}=1}^{\mathrm{m}} \mathrm{w}_{\mathrm{j}}^{2} \times R_{\mathrm{ij}}}{\sqrt{\sum_{\mathrm{j}=1}^{\mathrm{m}}\left(\mathrm{w}_{\mathrm{j}} R_{\mathrm{ij}}\right)^{2}} \times \sqrt{\sum_{\mathrm{j}=1}^{\mathrm{m}} \mathrm{w}_{\mathrm{j}}^{2}}}
\end{aligned}
$$

其中 $i=1,2 \cdots n$

将模的大小与夹角余弦的大小综 合考虑, 就可以全面准确的反应各个决 策方案与理想方案之间的接近程度。称 决策方案在理想方案上的投影值为灰 色关联投影值 $D_{i}$, 则有:

$$
\begin{aligned}
& D_{\mathrm{i}}=\left\|A_{\mathrm{i}}\right\| \times \cos \theta_{\mathrm{i}}=\left\|A_{\mathrm{i}}\right\| \times \frac{A_{\mathrm{i}} \times A_{0}}{\left\|A_{\mathrm{i}}\right\| \times\left\|A_{0}\right\|} \\
& =\frac{\sum_{\mathrm{j}=1}^{\mathrm{m}} R_{\mathrm{ij}} \times \mathrm{w}_{\mathrm{j}}^{2}}{\sqrt{\sum_{\mathrm{j}=1}^{\mathrm{m}} \mathrm{w}_{\mathrm{j}}^{2}}}=\sum_{\mathrm{j}=1}^{\mathrm{m}} R_{\mathrm{ij}} \times\left(\frac{\mathrm{w}_{\mathrm{j}}^{2}}{\sqrt{\sum_{\mathrm{j}=1}^{\mathrm{m}} \mathrm{w}_{\mathrm{j}}^{2}}}\right)
\end{aligned}
$$

（6）灰色关联投影值 $D_{i}$ 综合的反 映了决策方案 $A_{i}$ 与理想方案 $A_{0}$ 之间的 接近程度。最后根据 $D_{i}$ 的大小对各决策 方案进行排序。 $D_{i}$ 越大, 说明该方案与 理想方案与接近, 则该方案越优秀。

\section{4. 实证分析}

\section{1. 指标体系的建立}

综合考虑数据的可获得性、可操作 性以及科学性, 实证部分主要选取金融 总体规模、银行业、保险业以及证券业 
作为一级指标, 并相应的选取了 13 个 二级指标。如下表所示:

\begin{tabular}{|c|c|}
\hline 一级指标 & 二级指标 \\
\hline $\begin{array}{c}\text { 金融总体规 } \\
\text { 模 }\end{array}$ & \begin{tabular}{c|} 
金融业生产总值 \\
金融业区位商
\end{tabular} \\
\hline 银行业 & 年末金融机构存款余 \\
& 额 \\
& 年末金融机构贷款余 \\
& 额 \\
& 居民储蓄存款余额 \\
& 银行业区位商 \\
\hline & 年保费收入 \\
& 保险密度 \\
& 保险深度 \\
& 保险业区位商 \\
\hline & 上市公司数 \\
& 股票市价总值 \\
& 证券业区位商 \\
\hline
\end{tabular}

为了让数据更具有说服性, 本论文 的分析数据主要选取贵州省 9 个地州市 最近五年的数据, 并且对不同地州市的 同一年数据分别计算出每年的各决策 方案在理想方案上投影值的向量。其中 数据来源于中国保监会、贵州省统计局 以及各地州市统计官网和政府官网。其 中使用了 EXCEL 软件对每一年的各地州 市指标数据进行了处理。

\section{2. 数据的处理分析}

由于金融业生产总值数据的不可 获得性，所以本论文选取年末金融机构 存款余额、年保费收入以及股票市价总 值加总后的值作为金融业生产总值的 代替值。其中，根据贵州省各地州市近 五年数据可知最佳方案: $A_{0}=\{11962.2,3.27,9928.3,9153.2$, 2486.07,1.70,114.35,2434.64,0.0362,

\section{$2.97,16,4295.11,3.53\}$}

再根据模型建立部分的相关公式 得出 2012、2013、2014、2015、2016
年的决策方案在理想方案上投影值的 向量 $D$ 分别为:

$D(2012)=(0.02143,0.02017,0.03530$, $0.02017,0.02169,0.02056,0.02875$, $0.02018,0.02017)$

$D(2013)=(0.019760 .01863,0.02633$, $0.01862,0.01940,0.01915,0.02896$, $0.01864,0.01862)$ $D(2014)=(0.01876,0.01731,0.02514$, $0.01730,0.01805,0.01786,0.02859$, $0.01732,0.01731)$ $D(2015)=(0.02018,0.01823,0.02772$, $0.01821,0.01879,0.01904,0.03199$, $0.01823,0.01822)$

$D(2016)=(0.02251,0.02081,0.04402$, $0.02078,0.02140,0.02158,0.04197$, $0.02080,0.02079)$

为了更客观的评价各地州市的金 融集聚度，对同一地区不同年份的投影 值取均值得:

$\bar{D}=(0.02053,0.01903,0.03170$ ， $0.01902,0.01987,0.01964,0.03205$, $0.01903,0.01902$ )

下面对以上结果做一个折线统计 图。如下图所示:

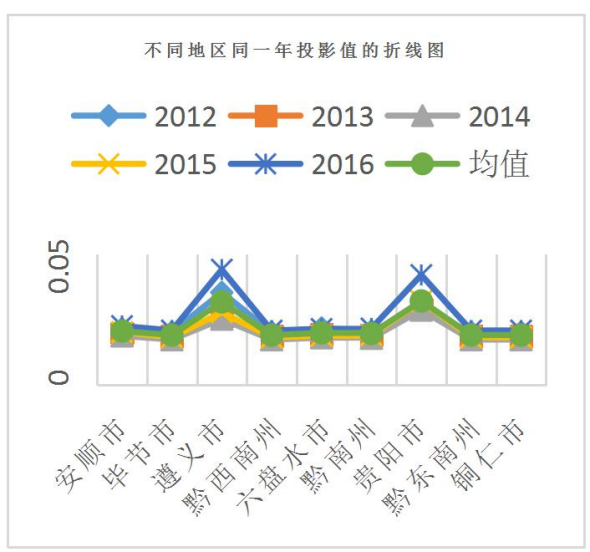


通过对以上各决策方案在理想方 案上的投影值大小比较以及从不同地 区投影值的折线图可以得出贵阳市、遵 义市每一年的投影值都比较大。从近五 年的投影均值中得知, 最高的是贵阳市, 为 0.03205 , 其次是遵义。而黔西南州 和铜仁市的金融集聚度最低, 与理想方 案相差大, 其中近五年来的投影均值为 0.01902 。

\section{5. 结论与讨论}

本研究建立了基于灰色关联投影 法的贵州金融集聚评价模型，并用其对 九个地州市的金融集聚程度进行了综 合评价。

本文的意义在于建立一种新的金 融集聚程度评价方法，并确定金融集聚 度的标准，这是灰色关联投影法面临的 首要问题, 而指标的确定和权重的确定 不是本研究的重点所在。然而灰色关于 投影法的不足之处在于投影值离散性 不强, 接下来的工作将加大观测密度和 增强了投影值的离散性，以提高金融集 聚度评价的精确度。

\section{参考文献}

[1] Wei Wang,Formal Finance, Informal Finance and Private Listed Enterprises' Bank Loan Defaults[J]. JRACR, 2015:1653-165.

[2] 李林, 丁艺, 李斌. 金融集聚与区域经 济增长关系研究 $[J]$. 统计与决 策,2009,6:131-134.

[3] Kindle Berger C.P., The Formation of Financial Centers: A Study of Comparative Economic History [M]. Princeton: Princeton University Press, 1974.

[4] 施蒂格勒（George J.Stigler）著,潘振 民译,产业组织与政府管制 $[M]$.上海: 上海三联出版社, 1989 .
[5] Feldman M.P. and Audretsch D.B. Innovation in Cities: Science based Diversity, Specialization and Localized Competition [J]. European Economics Review 43, 1999;409-429.

[6] Bossone B. Mahajan S. and Zahir F. Financial Infrastructure, Group Interests and Capital Accumulation [M]. IMF Working paper, 2003.

[7] 罗子嫄,何宜庆,毛华.华东地区金融 集聚与经济发展耦合关系研究 $[J]$. 企业经济,2013,8:135-138.

[8] 李静,白江. 我国地区金融集聚水平 的测度 $[J]$. 求是学刊,2014,4:52-58

[9] 邓薇,吕勇斌,赵琼.区域金融集聚评 价指标体系的构建与实证分析 $[J]$. 统计与决策,2015,19:153-155. 